\author{
COMMISSIONS G1 AND G4 OF THE IAU \\ INFORMATION BULLETIN ON VARIABLE STARS \\ Volume 62 Number 6194 DOI: 10.22444/IBVS.6194
}

Konkoly Observatory

Budapest

10 January 2017

HU ISSN $0374-0676$

\title{
SEARCH FOR VARIABILITY OF FIVE CENTRAL STARS OF PLANETARY NEBULAE
}

\author{
PAUNZEN, E. ${ }^{1} ;$ NETOPIL, M. ${ }^{1}$; RODE-PAUNZEN, M. ${ }^{2}$ \\ ${ }^{1}$ Department of Theoretical Physics and Astrophysics, Masaryk University, Kotlářská 2, 61137 Brno, Czech \\ Republic \\ ${ }^{2}$ Institut für Astrophysik der Universität Wien, Türkenschanzstr. 17, A-1180 Wien, Austria
}

\section{Introduction}

Planetary nebulae (PN) are the next-to-last stages of evolution of stars with main sequence masses between 0.8 and $8 M_{\odot}$. As the core slowly collapses, the outer layers are ejected forming the typical nebula-like structures. Nowadays it is beyond doubt that there is a connection between binary central stars and PN shaping (Hillwig et al. 2016). However, due to projection effects, even a spherical symmetric shape does not imply an apparent single star nature. It is therefore important to search for signs of binarity using photometric time series. One would either expect classical eclipses or reflection effects due to the orbital motion of the components.

Another type of variability is attributed to variations in the stellar wind, but also pulsation of some objects has been suggested as a possible explanation (Handler et al. 1997). These variations have been found in all type of PN central stars. For example, HD 35914, the variable central star of the PN IC 418 shows two distinct kinds of variability: irregular light modulation with a time scale of days (amplitude of about $0.3 \mathrm{mag}$ ), as well as cyclic variations with a time scale of hours (0.02 mag).

De Marco et al. (2008) summarized the back then known variability properties of PNe (Figure 4 therein) including all above described mechanisms. The observed periods range up to three days with amplitudes up to two magnitudes.

Here, we present photometric observations of five central stars of PNe, Kronberger PN J1944.9+2245, NGC 6853, NGC 7008, NGC 7076, and NGC 7354 in order to search for variability on time scale from hours to days. We were not able to detect any signs of variability with upper limits between 0.01 and $0.12 \mathrm{mag}$, respectively.

\section{Observations and data reduction}

The observations were performed in August and September 2010 at the Hvar Observatory, University of Zagreb (Croatia), using the $1 \mathrm{~m}$ Austrian-Croatian Telescope (ACT). The 
Table 1: Observation log and results. Kronberger PN J1944.9+2245 was also investigated for variations in four nights on the time scale of hours (second line).

\begin{tabular}{lccccc}
\hline PN & $\begin{array}{c}V \\
{[\mathrm{mag}]}\end{array}$ & $\begin{array}{c}\text { HJD(start) } \\
2455792+\end{array}$ & $\begin{array}{c}\text { HJD(end) } \\
2455792+\end{array}$ & $N$ & $\begin{array}{c}\text { Upper Limit } \\
{[\mathrm{mag}]}\end{array}$ \\
\hline J1944.9+2245 & 15.8 & 0.44241 & 10.37849 & 42 & 0.12 \\
J1944.9+2245 & & 44.25059 & 49.27524 & 131 & 0.10 \\
NGC 6853 & 14.1 & 0.45868 & 45.31385 & 52 & 0.08 \\
NGC 7008 & 12.8 & 0.47488 & 49.40449 & 64 & 0.01 \\
NGC 7076 & 13.4 & 0.48364 & 44.41168 & 38 & 0.06 \\
NGC 7354 & 16.1 & 0.49543 & 49.42055 & 44 & 0.07 \\
\hline
\end{tabular}

telescope was equipped with an Apogee Alta U47 CCD camera of $1024 \times 1024$ pixels, resulting in a field-of-view of about $3^{\prime}$ square. The integration times for the observations in the Bessell $I$ filter system were set between 45 and $120 \mathrm{~s}$, depending on the weather conditions and the brightness of the target. After the basic CCD reductions (bias-subtraction and flat fielding), we applied aperture photometry within IRAF because all targets are in non-crowded fields. The sizes of the applied apertures were inspected manually for all observations to minimize the effects of the surrounding nebula. The further reduction steps were performed using the standard technique for time series CCD observations.

In each field, there were at least seven comparison stars of similar brightness as the target. The differential light curves derived from at least five comparison stars within the field were used for the time-series analysis. This should guarantee eliminating possible unknown instrumental effects. All differential light curves were examined in more detail using the Phase-Dispersion-Minimization method (PDM) within the software Peranso (Paunzen \& Vanmunster 2016). An analysis with a discrete Fourier algorithm gave the same noise level over the searched frequency range as PDM.

\section{Analysis and conclusions}

For all five targets, we searched for long-term variations on the time scales of days. For this, three to five consecutive observations in one night were secured. The time-series analysis was performed using all individual measurements, but also for the mean of all individual nights. In Table 1 , the results are summarized. We were not able to detect any statistically significant ( $4 \sigma$ above the noise level) signal in the light curves. The upper limits range from 0.01 to $0.12 \mathrm{mag}$. Hillwig et al. (2015) presented variability caused by binarity for the central star of Abell 65. The amplitude in the $I$ filter for this object is about one magnitude with a period very close to one day. In addition, the characteristics for other known binaries among PN central stars are very similar (De Marco et al. 2008). Therefore we conclude that none of the investigated objects shows eclipses and irradiation effects on the investigated time scale of up to 50 days.

The object Kronberger PN J1944.9+2245 was also investigated during four nights searching for variation on a time scale up to a few hours. The noise level is rather high (0.1 mag) compared to the cyclic variations (0.02 mag) found by Handler et al. (1997) for the central star of IC 418. We can therefore not exclude such variations for this object.

We encourage further photometric observations of PN central stars to detect to-date unknown binary systems which is a perfect suited project for smaller telescopes. The 
expected variations can reach even up to a few magnitudes which is observable in nonperfect conditions and sites. With the prospects of precise parallaxes and thus distances from the Gaia, such observations will significantly contribute to our knowledge of PNe.

Acknowledgements: The work was supported by the Czech Grant Agency under the project $14-26115 \mathrm{P}$.

References:

De Marco, O., Hillwig, T. C., Smith, A. J., 2008, AJ, 136, 323 DOI

Handler, G., et al., 1997, $A \& A$, 320, 125

Hillwig, T. C., et al., 2015, $A J, \mathbf{1 5 0}, 30$ DOI

Hillwig, T. C., et al., 2016, ApJ, 832, 125 DOI

Paunzen, E., Vanmunster, T., 2016, AN, 337, 239 DOI 